\title{
Electrical model of carbon fibre reinforced polymers for the development of electrical protection systems for more-electric aircraft
}

\author{
C.E. Jones, P.J. Norman, \\ S.J. Galloway, G.M. Burt \\ Department of Electronics and Electrical \\ Engineering \\ UNIVERSITY OF STRATHCLYDE \\ 204 George Street \\ Glasgow, UK G1 1XW \\ E-Mail: catherine.e.jones@strath.ac.uk
}

\author{
L.F. Kawashita, M.I. Jones, \\ S.R. Hallett \\ Advanced Composites Centre for Innovation \\ and Science \\ UNIVERSITY OF BRISTOL \\ Queen's Building, University Walk \\ Bristol, UK BS8 1TR \\ E-Mail: Stephen.hallett@bristol.ac.uk
}

\section{Acknowledgements}

This work has been undertaken within the Rolls-Royce University Technology Centres for Electrical Power Systems and Composite Materials.

\section{Keywords}

«Airplane », «Fault handling strategy », «Fault ride-through », «Fault tolerance »

\begin{abstract}
Carbon fibre reinforced polymers (CFRP) are increasingly used for structures on aircraft due to their superior mechanical properties compared to traditional materials, such as aluminium. Additionally, in order to improve aircraft performance, there is a continued trend for electrically driven loads on aircraft, increasing the on-board electrical power generation capacity and complexity of the electrical power system, including a desire to increase voltage levels and move towards DC distribution systems.

Central to the reliable operation of an electrical power system is the development of an appropriate protection and fault management strategy. If an electrical earth fault occurs on a composite moreelectric aircraft then the CFRP may form part of the route to ground. In order to develop an appropriate protection system and thus to understand the effects on engine generators it is necessary to investigate the fault response of this network. Hence a suitable electrical model of the CFRP material is required, which will enable CFRP to be included in a computationally-intensive systems-level simulation study of a more-electric aircraft (MEA) with fully switching power electronic converter models.

This paper presents an experimentally validated impedance model of CFRP at an appropriate level of fidelity for use in systems level simulation platforms, enabling appropriate protection methods to be developed. The validated model considers the impact of the electrical bonding to ground, including the impedance added by a metallic frame that a CFRP panel may be mounted in. The simplicity of the model results in a less complex process to determine the expected impedance of the CFRP material, enabling a focus on the fault response of the system and subsequent development of appropriate protection solutions.
\end{abstract}




\section{Introduction}

The continued trend for increasing commercial air travel, coupled with a desire to reduce the environmental impact of aircraft has provided strong motivation for the continued development of the MEA [1]. As a result, the levels of on-board electrical power generation that is primarily supplied by the engine generators will increase as the loads, traditionally powered mechanically, hydraulically or pneumatically, are increasingly driven instead by electrical power [1-3]. Consequently, this results in a trend for more complex, on-board aero-electrical power systems. Power electronics are a key enabling technology for these aero-electrical power systems, allowing a variety of voltage levels, frequencies, alternating and direct currents, and loads to be supplied [2]. Alongside this, there is an upwards trend for the use of CFRP due to the superior mechanical properties that such materials have compared to more traditional materials, such as high grade aluminium alloys [4]. More than $50 \%$ of the empty weight of current state of the art aircraft are constructed from composite materials, the majority of which are laminated carbon fibre reinforced polymers (CFRP) $[5,6]$.

It is well documented that the increased use of CFRP presents a significant challenge electrically $[7,8,10]$ due to its poor electrical conductivity properties compared to aluminium. Hence such aircraft commonly require the installation of a metallic current return network [4,11]. However, if a ground fault should occur, due for example to a chafed wire [12], there is a possibility that the fault current may require to pass through the CFRP material to reach the ground return network [7]. This may affect the fault response of the system, and conventional fault detection methods may no longer be appropriate. Therefore an estimation of the impedance of the CFRP material is required to enable appropriate fault management and electrical protection system design.

Existing numerical models of CFRP materials presented in the literature are inappropriate for use in circuit simulations for protection system design as they require a full power system simulation $[7,13]$ due to their high level of numerical complexity. In addition, the impact of the method of electrical bonding to ground is not considered but is expected to be a critical feature in protection studies. This paper will present the development of an appropriate modelling concept, and demonstrate its suitability for use in systems level studies.

\section{State of the art aircraft protection challenges}

\section{State-of-the-art MEA power system design}

The key driver behind the development of composite, MEA aircraft is the desire to minimise fuel burn to improve overall system performance. A holistic approach must be taken to develop the aeroelectrical power system, to ensure that it is designed and developed to meet required performance criteria and relevant standards. The development of an appropriate protection and fault management system for a more-electric aircraft is influenced by (and will itself influence) the power system architecture (including power generation), and reliability and redundancy considerations, and also by external factors (including the material that the aircraft is made from). Ultimately, these four interdependent elements will all impact on the performance (fuel burn) of the aircraft.

\section{State-of-the-art more-electric architectures and protection considerations}

Recent MEA architectures have included the development of remote distribution, where distribution centres are placed closer to loads, reducing cable losses and weight, reducing both lengths of cable and cable power ratings [14]. Linked to this, there is a trend for higher distribution voltages (540 VDC, 230 Vph RMS), reducing distribution cable current ratings, and further reducing fuel burn. A key 
enabling technology of the MEA electrical architecture is power electronics [2]. Power electronic converters are required to enable a constant voltage, variable frequency electrical power system to operate, enabling electrical machines to run at optimal speeds, improving system performance and efficiency.

To develop an appropriate protection and fault management system, the fault response of a ground fault on a composite aircraft requires to be understood. The key design criteria for an electrical protection system include reliability, selectivity, stability, speed and sensitivity [15]. An additional consideration for an aero-electrical power system application would be the weight penalty attributed to the protection system [16].

If the fault path of a ground fault current is through a section of CFRP, then this may become a high impedance fault, impacting on fault response and hence the protection and fault management strategies. An example section of aero-electrical network is shown in Figure 1, where the voltage source converter interfaced section of DC network has a + 270 VDC rail/cable-to-ground fault through a section of CFRP material. In Figure 1 the CFRP impedance is $Z_{c}(\Omega)$, the impedance of the DC section of the network, which may include filters, is $Z_{n e t}(\Omega)$, and the impedance of the current return network, which may require to be considered is $Z_{g n d}(\Omega)$. The impedance $Z_{g n d}(\Omega)$ is expected to be 2 $\mathrm{m} \Omega / \mathrm{m}$ and $8 \mu \mathrm{H} / \mathrm{m}[17]$.

The first step to developing an understanding of the fault response is to develop an electrical model of CFRP material with an appropriate level of modelling fidelity for use within systems level studies. To date the electrical characterisation of CFRP materials has been conducted at a detailed level for material model development [7]. These models are too numerically complex (and hence computationally intensive) to be suitable for inclusion in a systems level study of an aero-electrical power system, which will require the modelling of a complex electrical power system including full, switching models of power electronic converters.

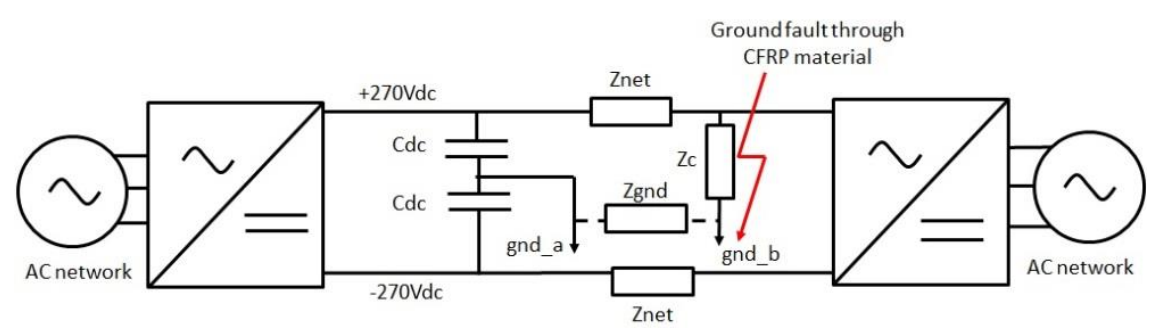

Figure 1: Example system with a +270 VDC rail to ground fault through CFRP material.

\section{Electrical impedance model of CFRP}

\section{Overview of CFRP material}

CFRP laminate materials are made up from layers, referred to as plies, of carbon fibres, with a resin holding the carbon fibres in a particular arrangement and transferring mechanical loads to the carbon fibre [18]. The fibres in each ply can either be woven or non-woven (unidirectional). The orientation of the carbon fibres is often varied between plies, to create the desired mechanical stiffness and strength.

From an electrical perspective, the carbon fibre has a high electrical conductivity of $40-50 \mathrm{kS} / \mathrm{m}$, but the resin is considered to be an electrical insulator [19]. The wavy nature of carbon fibres, due to imperfections occurring during the manufacturing process or degradation of the material, may 
cause fibres to touch both between plies and within the same ply. Hence CFRP cannot be considered to be an electrical insulator.
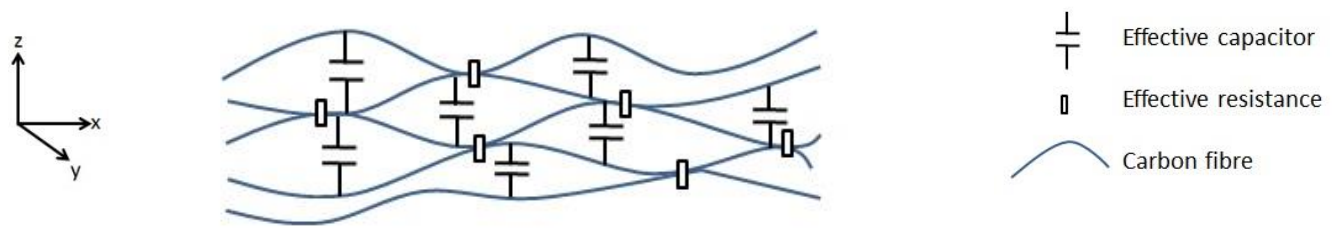

Figure 2: Simplified 2-dimensional diagram of the $x$ (along fibre) and $z$ (transverse) electrical model of a section of unidirectional CFRP.

Due to the non-uniform nature of the electrical connections between fibres, the impedance of the model forms a highly complex lattice arrangement. It has been proposed by [20] that the gaps between fibres filled with epoxy resin will result in the impedance of the material have a capacitive characteristic in the out-of-plane transverse $(z)$ and in-plane transverse $(y)$ directions, and purely resistive characteristic in the along fibre $(x)$ direction. A simplified diagram of the material in 2dimensions is shown in Figure 2. In practice there would also be electrical connections in the $y$ direction. Due to the high unpredictability of these electrical characteristics, at a high level of detail the material is extremely complex from both an electrical characterisation perspective and the unpredictability of these electrical connections.

\section{Proposed modelling concept}

As a starting point to develop a model of CFRP with the desired level of fidelity, consideration was given to how electrical current would flow through the material to ground under a fault condition. A key assumption made is that the CFRP is mounted in a metallic frame, and that that frame is solidly bonded to electrical ground. For the model developed it is assumed that one side of the frame is connected, however the model can be adapted for different orientations and numbers of sides connected to ground. Due to the highly complex nature of multi-directional laminated composites, the initial model was developed for unidirectional $\left[0^{\circ}\right]$ material. In this case, Hexcel's IM7/8552 carbonfibre/epoxy pre-preg system [22].

The modelling concept presented assumes that an electrical current, which enters through the top ply of a CFRP laminate, will take the path of least electrical resistance to ground. Therefore, it is proposed (Figure 3) that after the electrical fault current has passed through the surface epoxy layer of the CFRP, it will flow along the direction of the carbon fibres to the edge of the material. If necessary, fault current will then flow around the edge of the material to the connection to ground. The key advantage of this approach is that the CFRP can be modelled as a lumped impedance network, as shown in Figure 3, which minimises the computation overhead such a model would place on a systems level simulation. It is assumed that due to the fibre orientation, the impedance of the material in the $x$-direction will be much lower than the $y$-direction, and hence no current will flow in the $y$-direction to the edge of the CFRP.

In order to estimate the values of the impedances, it is proposed that if the conductivity, $\sigma(\mathrm{S} / \mathrm{m})$ (for $z$ - and $x$-directions) and dielectric permittivity, $\varepsilon_{r}$, (for the $z$-direction) are known, then the equivalent impedances can be calculated using (1) and (2), where $A\left(\mathrm{~m}^{2}\right)$ is cross-sectional area, $l$ is distance $(\mathrm{m})$ and $\varepsilon_{0}(\mathrm{~F} / \mathrm{m})$ is the dielectric constant.

$$
R_{x, z}=\frac{1}{A_{x, z} \sigma_{x, z}} l_{x, z}
$$




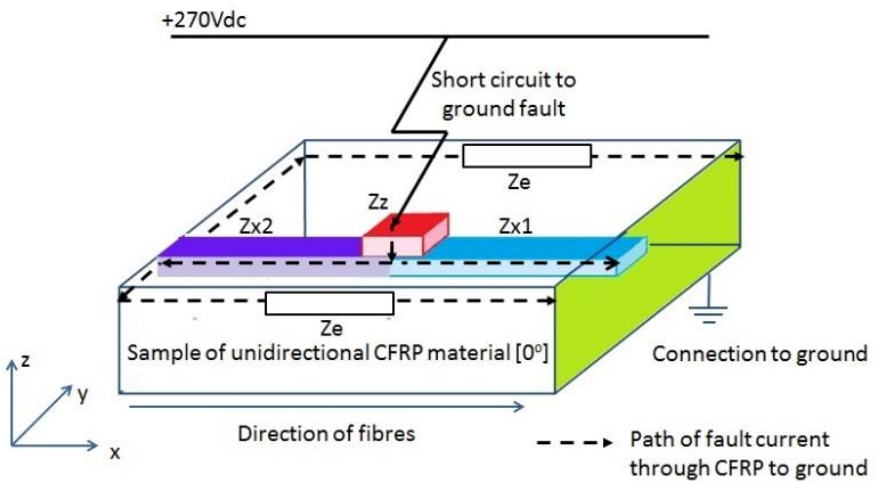

(a)

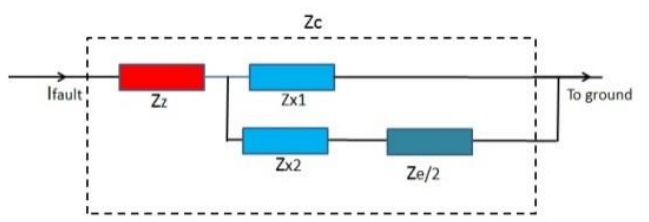

(b)

Figure 3: Example of the proposed electrical model. Proposed route of fault current through the material to ground (a) and the equivalent electrical circuit diagram (b), where $Z_{z}(\Omega)$ is the impedance of the material in the $z$-direction, $Z_{x l}(\Omega)$ and $Z_{x 2}(\Omega)$ the impedance in the $x$-direction and $Z_{e}$ is the impedance of the edge of the material.

\section{Experimental validation of modelling concept}

\section{Experimental measurement of conductivity}

To validate this modelling concept, the first step was to estimate the conductivity of the CFRP material. From the literature, it is known that the conductivity of the CFRP material varies linearly with distance in the direction of the fibres ( $x$-direction) [9]. The variation of the electrical resistance, $R$, in the $x$-direction with distance can be expressed using (3), where $A$ is the cross-sectional area of the sample $\left(\mathrm{m}^{2}\right), L$ is the length $(\mathrm{m}), \sigma$ is electrical conductivity $(\mathrm{S} / \mathrm{m})$ and $R_{c}$ is contact resistance $(\Omega)$.

$$
R=\frac{1}{A \sigma} L+R_{C}
$$

The resistance of a number of CFRP samples of lengths from 25 to $100 \mathrm{~mm}$ was measured. The thickness of the samples was 40 plies $(5 \mathrm{~mm})$ and the width was $10 \mathrm{~mm}$. In order to minimise contact resistance, the ends of the samples were polished using sandpaper and silver paint was applied. A copper foil electrode was then placed on the silver paint, and pressure was applied to the ends of the sample using a vice. Kelvin probes were used with an LCR meter to further reduce contact resistance. The experimental set up in the laboratory is shown in Figure 4, there is a small amount of variation in the value of $\sigma_{x}$ between samples, due to the non-uniform nature of CFRP material. In the $x$-direction, the electrical model of CFRP is considered to be purely resistive, as it is assumed that the electrical current will flow along unbroken, electrical conductors to the edge of the CFRP panel.

The $y$-direction conductivity was measured to be $3 \mathrm{~S} / \mathrm{m}$. As this is significantly lower than the conductivity in the $x$-direction, it is assumed that all the electrical current will flow along the direction of the fibres to the metallic edge of the panel. Hence the impedance in the $y$-direction was not investigated further or variation with distance considered.

As presented in Figure 2, the impedance of the material in the $z$-direction is expected to be complex and represented as a parallel resistive-capacitive circuit Figure 5. This impedance can be represented in the $s$-domain as a first order system (4) with a cut-off frequency given by (6). If the capacitance can 
be estimated, then the dielectric constant for the material can be calculated using (5) and used to estimate the capacitance of a section of CFRP panel.

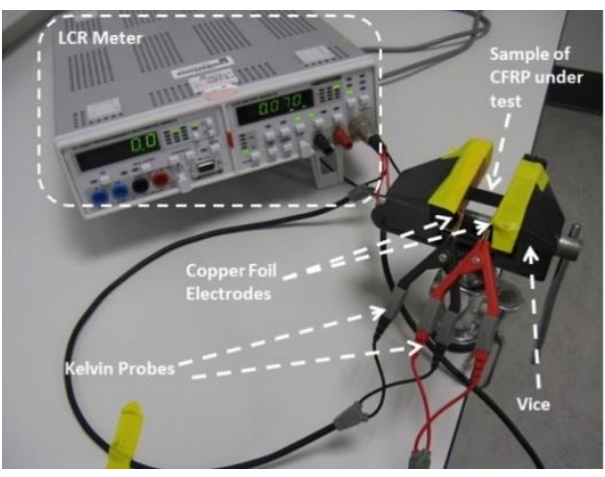

(a)

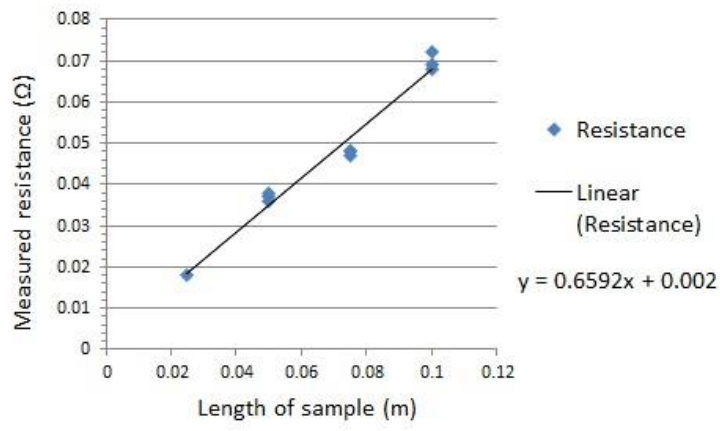

(b)

Figure 4: Experimental method for conductivity measurement (a), measured resistance in the $x$-direction (b).

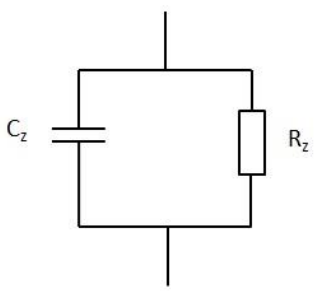

(a)

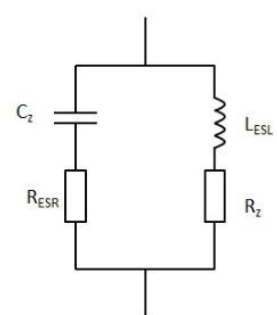

(b)

Figure 5: Equivalent resistive capacitive network (a) and equivalent resistive capacitive network with parasitic inductance and resistance (b).

$$
\begin{aligned}
& Z_{z}(s)=\frac{R_{Z}}{1+s R_{z} C_{z}} \\
& C=\frac{\varepsilon_{0} \varepsilon_{r} A}{d} \\
& f_{01}=\frac{1}{2 \pi C_{z} R_{z}} \\
& f_{02}=\frac{1}{2 \pi} \sqrt{\frac{1}{C_{z} L_{z}}}
\end{aligned}
$$

The frequency response of a $25 \times 10 \times 5 \mathrm{~mm}$ sample of unidirectional CFRP was measured using a spectrum analyser, over the range of $0.1-100 \mathrm{kHz}$. By choosing values for the system parameters as indicated in Table I, then the frequency response for the parallel R-C system can be determined (as shown in blue in Figure 6). If the model in Figure 5 (a) is extended as shown in Figure 5 (b) to include parasitic inductance in series with the resistance, $L_{E S L}(\mathrm{H})$, and a small amount of parasitic resistance in series with the capacitance, $R_{E S R}(\Omega)$, then this results in a second order system, which has a cut off frequency given by (7). This gives a better fit to the experimental results, as indicated in Figure 6. 
For initial fault studies and preliminary protection system design, the reduced order R-C parallel circuit model provides a sufficient level of detail but minimises the overall simulation computational overhead, and has been utilised for the remainder of the paper. The more detailed model configurations will be employed in later studies to validate the findings of the reduced order model. The dielectric constant of the material was found to be 7.34 using (5). This is comparable to values published in the literature [21]. The conductivity is calculated to be $0.8 \mathrm{~S} / \mathrm{m}$ from (1). The variation with distance was not investigated as the thickness of the panel is unlikely to vary significantly.

Table I: System impedance parameters

\begin{tabular}{|l|l|l|}
\hline Parameter & Parallel Resistor-capacitor & Parallel Resistor with $L_{E S L}$ - Capacitor with $R_{E S R}$ \\
\hline$R_{z}(\Omega)$ & 21.8 & 21.8 \\
\hline$C_{z}(\mathrm{pF})$ & 3.25 & 3.9 \\
\hline$L_{E S L}(\mathrm{nH})$ & - & 0.25 \\
\hline$R_{E S R}(\mathrm{~m} \Omega)$ & - & 1 \\
\hline$f_{0}(\mathrm{GHz})$ & 2.22 & 5.09 \\
\hline
\end{tabular}

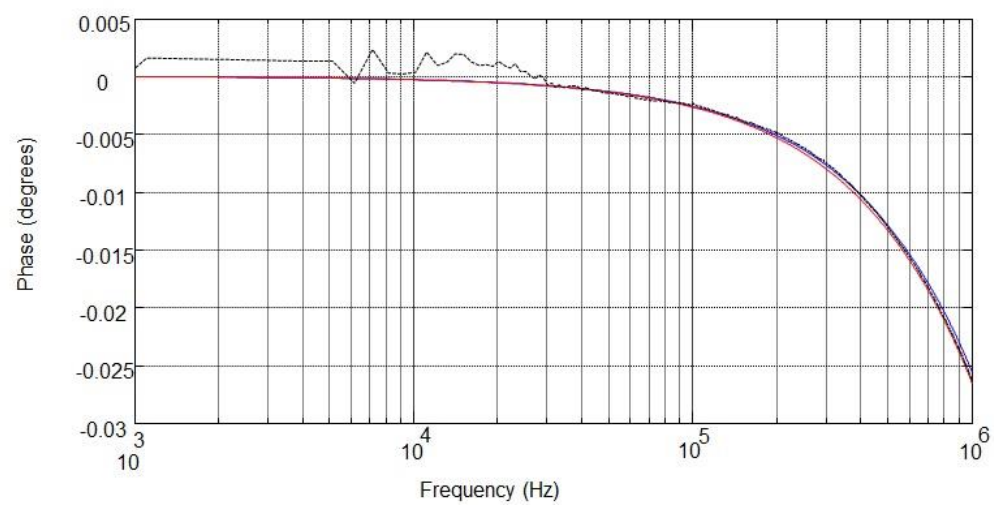

Figure 6: Comparison of theoretical and experimental measured frequency response of the impedance in the $z$-direction.

\section{Consideration of electrical bonding to ground}

In Figure 3 the electrical resistance of the edge of the material, $R e(\Omega)$, includes the frame and any adhesive bonding materials. The impact of any adhesive bonding materials on the electrical characteristics of the material has not been considered any further at this stage. Whilst the electrical conductivity of the metallic edge of the material may be relatively high in comparison to that of the CFRP itself [23], this aspect of the structure must be considered in the electrical model. Firstly any additional materials used to bond the CFRP to the metal frame may impact on the overall impedance. Secondly, whilst the impedance may be negligible for some fault locations and scenarios, it may not be for others.

\section{Experimental validation}

In order to validate the modelling concept, the impedance of a $100 \times 100 \times 5 \mathrm{~mm}$ panel of CFRP when a fault is applied to an area of $10 \times 10 \mathrm{~mm}$ on the surface of the material, was measured. A number of $10 \times 10 \mathrm{~mm}$ electrodes were placed on the surface of the sample, as indicated in Figure 7 . The test was conducted with the area under the electrodes polished and un-polished. In order to achieve a conductive edge to the material, silver paint was applied to the edges of the CFRP panel, with copper foil applied at the corners to ensure good electrical conductivity around the material. The value of the 
edge resistance, $R e$, was $5.24 \Omega$. For the un-polished samples, it was found that the electrical current flowed a distance of 1 ply $(0.125 \mathrm{~mm})$ in the $z$-direction into the CFRP, and over a depth of 1.2 plies $(0.15 \mathrm{~mm})$ to the edge of the material. The total CFRP resistance was calculated using a combination of (1) (to obtain values for the individual resistances) and (8). For the polished samples, the value of $R_{z}$ was zero, due to the removal of the top layer of epoxy from the CFRP sample. The calculated theoretical values in Table II give generally good correlation with the experimentally measured values. It is expected that there will be differences between measurements due to the irregular nature of the CFRP material.

$$
R_{T}=R_{z}+\frac{R_{x 1}\left(R_{x 2}+\frac{R_{e}}{2}\right)}{R_{x 1}+R_{x 2}+\frac{R_{e}}{2}}
$$

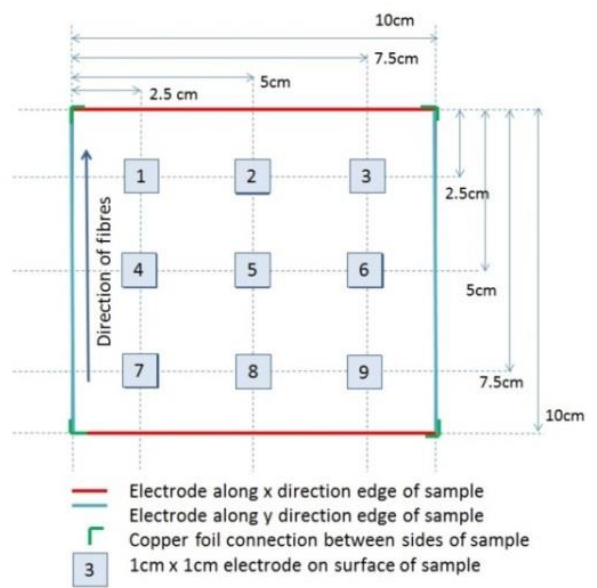

Figure 7: Preparation of CFRP panel for validation tests.

Table II: Comparison of theoretical and experimental results

\begin{tabular}{|l|l|l|l|r|}
\hline $\begin{array}{l}\text { Electrode } \\
\text { number }\end{array}$ & \multicolumn{2}{|l|}{ Polished Case } & \multicolumn{2}{l|}{ Unpolished case } \\
\hline & $\begin{array}{l}\text { Theoretical } \\
\text { Resistance } \\
(\Omega)\end{array}$ & $\begin{array}{l}\text { Measured } \\
\text { resistance } \\
(\Omega)\end{array}$ & $\begin{array}{l}\text { Theoretical } \\
\text { Resistance } \\
(\Omega)\end{array}$ & $\begin{array}{l}\text { Measured } \\
\text { resistance } \\
(\Omega)\end{array}$ \\
\hline 1 & 0.431 & 0.484 & 1.997 & 2.881 \\
\hline 2 & 0.431 & 0.451 & 1.997 & 2.191 \\
\hline 3 & 0.431 & 0.6 & 1.997 & 1.5 \\
\hline 4 & 0.627 & 0.787 & 2.200 & 3.345 \\
\hline 5 & 0.627 & 0.577 & 2.200 & 2.28 \\
\hline 6 & 0.627 & 0.236 & 2.200 & 3.58 \\
\hline 7 & 0.588 & 0.604 & 2.173 & 1.8 \\
\hline 8 & 0.588 & 0.467 & 2.173 & 1.802 \\
\hline 9 & 0.588 & 0.741 & 2.173 & 0.3 \\
\hline
\end{tabular}

\section{Discussion}

The results presented in Table II indicate that, even over a short distance, the impedance added to the fault path by the CFRP material is significant, and that the fault can no longer be considered low-impedance. It is expected that in the real case the distance that a fault current may have to travel to reach the current return network may be much further. Indications from the literature and standards suggest that it would not be unreasonable for there to be a distance of at least $0.65 \mathrm{~m}$ between 
electrical bonding to ground on a panel of CFRP [24,25]. By inspection of (1) it is clear that the equivalent impedance of the CFRP material will also increase as the surface area that fault current enters a CFRP panel through is decreased. For example, if a panel is assumed to have dimensions of $0.65 \times 0.65 \mathrm{~m}$, and $10 \%$ of the circumference of an 0 AWG cable comes into contact with the CFRP panel $(2.57 \mathrm{~mm})$ over a length of $5-20 \mathrm{~mm}$, then the resistance will vary from circa 20 to $100 \Omega$.

By way of example, the proposed lumped impedance model of the CFRP enables the fault response of the scenario shown in Figure 1 to be investigated. With a low impedance fault $(0.01 \Omega)$ a high fault current is observed. This could be detected using conventional overcurrent methods. However, if the impedance is set to levels attributable to CFRP, ranging from 20 to $100 \Omega$, then the fault current is much lower. This not only indicates the need for developing new fault detection methods, but it changes the objective of the aero-electrical protection system. Conventionally, electrical protection is required to protect the healthy areas of the electrical network. In contrast, the levels of fault current through CFRP may not affect the operation of the electrical network, but will result in a current flowing through the CFRP material, which may lead to Joule heating and degradation of the structural material of the aircraft.

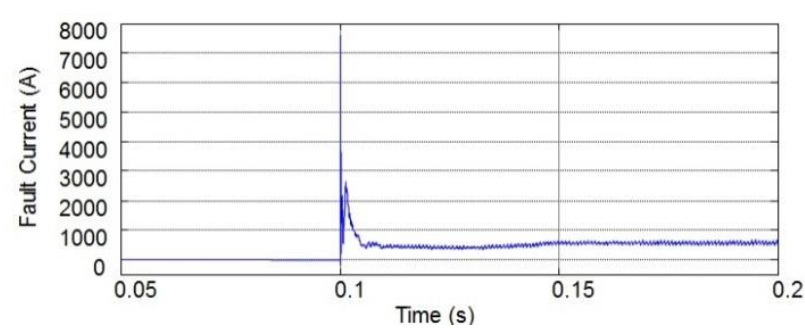

(a)

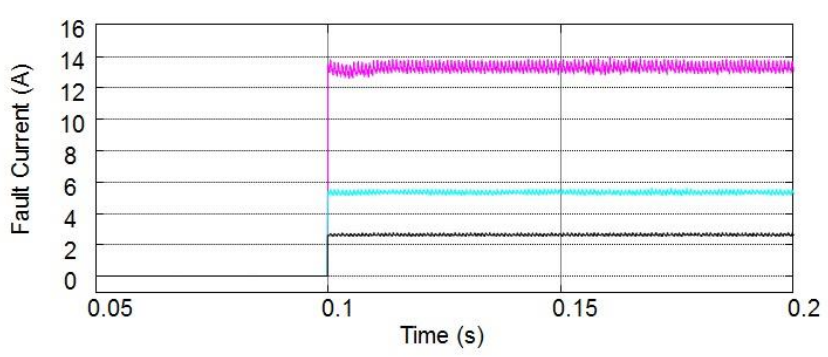

(b)

Figure 8: Fault response at $0.01 \Omega$ (a) and at $20 \Omega$ (magenta), $50 \Omega$ (cyan) and $100 \Omega$ (black) (b).

\section{Conclusions and future work}

The simplified, lumped impedance model proposed for CFRP is suitable for use within aero-electrical systems simulation platforms. This will enable the impact of CFRP on different ground fault scenarios to be investigated, in particular to focus the study on the fault response and development of appropriate protection and fault management strategies for increasingly complex aero-electrical networks. It is clear from the model developed that the contribution to the fault impedance by the CFRP is highly variable from fault to fault, including dependence on the location of the fault on a panel, panel dimensions, contact area and grounding methods used. The model developed will enable the further investigation of these variables on the fault response.

It is acknowledged that the model is thus far only developed for a single type of unidirectional $\left[0^{\circ}\right]$ CFRP laminate. The authors plan to extend the model to more complex multi-directional CFRP laminates in the future. The impact of the addition of conductive additives, such as carbon nanotubes must also be considered.

\section{References}

1. W. Cao. B. C. Mecrow, G.J. Atkinson et al, "Overview of electric motor technologies used for more electric aircraft", IEEE transactions on industrial electronics, Vol. 59, no. 9. Pages 3523 -3531, 2012.

2. P. Wheeler, S. Bozhko, "The more electric aircraft: technology and challenges", IEEE Electrification Magazine, Vol. 2, Issue 4, Pages 6 -12, 2014. 
3. B. Sarlioglu and C. T. Morris, "More electric aircraft: review, challenges and opportunities for commercial transport aircraft". IEEE Transactions on Transportation Electrification, Vol. 1, No. 1, Pages 54-64, 2015.

4. A.-L. Goleanu, M. Dunand, J.-M. Guichon et al, "Towards the conception and optimisation of the current return path in a composite aircraft", Annual IEEE System Conference, Pages 466-471, 2010

5. Airbus Group, "Taking the lead: the A350XWB”, 2006.

6. The Boeing Company, "Boeing 787 From the ground up", Boeing AERO magazine, QTR_4, 2006.

7. A. Piche et al, "Numerical modelling support for the understanding of current distribution in carbon fibers composites", International Symposium on Electromagnetic Compatibility, 2009.

8. A. Piche et al, "Dynamic electrical behaviour of a composite material during a short circuit", International Symposium on Electromagnetic Compatibility, 2011.

9. J.C. Abry et al, "In-situ monitoring of damage in CFRP laminates by means of AC and DC measurements", Composites Science and Technology, No. 61, Pages 855 - 864, 2001.

10. P. Ballocchi, M. Fogel, P. Parlevliet et al, "Enhancement of electrical conductivity of aerospace structures by incorporation of CNT doped carrier materials into dry preforms", 8 th International Technical Conference and Forum, SAMPE EUROPE SETEC 13, 2013.

11. Airbus Group, “A350 XWB Electrical Structure Network”, Flight Airworthiness Support Technology Airbus technical Magazine, Number 53, Pages 20 -24, 2014.

12. J. Rivenc et al, "Methodology for predicting degradation shape and depth of a CFRP during shortcircuit current injection”, More Electric Aircraft Conference (Toulouse, France), 2015.

13. J. Park et al, "Experimental and numerical study of the electrical anisotropy in unidirectional carbon fiber reinforced polymer composites”, Smart materials and structures, Vol. 16, pages 57-66, 2007.

14. The Boeing Company, "787 No-bleed systems", Aero Magazine, Quarter 4, 2007.

15. S. Fletcher, "Protection of physically compact multiterminal DC power systems", Doctoral Thesis, University of Strathclyde, 2013.

16. S. Fletcher, P. Norman, S. Galloway et al, "Modeling and simulation enabled UAV electrical power system design", SAE International Journal of Aerospace, 2011

17. M. Terörde, H, Wattar and D. Schulz, "Phase balancing for aircraft electrical distribution systems", IEEE Transactions on Aerospace and Electronic Systems, Vol. 51, Issue 3, Pages 1781-1792, 2015.

18. "HexPly prepreg technology", Hexcel Corporation, accessible online: http://www.hexcel.com/Resources/DataSheets/Brochure-Data-Sheets/Prepreg_Technology.pdf, website accessed April 2015.

19. L. Guadagno et al, "Effective formulation and processing of nanofilled carbon fiber reinforced composites", Royal Society of Chemistry Advances, No.5, Pages 6033 - 6042, 2015.

20. H. Wittich et al, "The measurement of electrical properties of CFRP for damage detection and strain recording", Proceedings of the second European conference on composite testing and standardisation (ECCM-CTS), Pages 447 - 457, 1994.

21. Z. M. Elimat et al, "Dielectric properties of epoxy/short carbon fibres", Journal of Material Science, Vol. 45, Pages 5196 - 5203, 2010.

22. Hexcel, "HexPly 8552", available online http://www.hexcel.com/Resources/DataSheets/Prepreg-DataSheets/8552_eu.pdf

23. Tibtech, "Properties of stainless steel, metals and other conductive materials" Available online: http://www.tibtech.com/conductivity.php, accessed May 2016.

24. British Standards Institute, "Aerospace series - Design and installation of aircraft electrical and optical interconnection systems”, BS EN 3197:2010, BSI Standards Publication, Dec. 2010.

25. R. Perraud et al, "Metallic strip details for validation of ESN technologies", Chapter 10, Smart Intelligent Aircraft Structures, Springer International Publishing, Switzerland, pages 839-856, September 2015 